\title{
EFFECT OF ROSMARINIC ACID AS A CHEMOPREVENTIVE MODALITY ON EXPERIMENTALLY INDUCED HAMSTER BUCCAL POUCH CARCINOGENESIS
}

\author{
Amr Saad Abd Al-Wahab *, Hany Gameil Gobran** \\ and Hesham Ahmed Hassan Dameer**
}

\begin{abstract}
Aim: It was directed to evaluate rosmarinic acid (RA) effect as a chemopreventive modality on experimentally induced hamster buccal pouch (HBP) carcinogenesis.

Material and methods: Forty Syrian male hamsters five weeks old, weighing 120-80g, were divided into four groups of ten hamsters in each as follows, GI: Only topical application of liquid paraffin ( 3 times a week for 14 weeks), GII: Topical application of 12,7 dimethyl benz[a]anthracene (DMBA) alone (\%0.5 in liquid paraffin, 3 times a week for 14 weeks), GIII: Topical application of DMBA (\%0.5 in liquid paraffin, 3 times a week for 14 weeks) associated by oral administration of RA $(100 \mathrm{mg} / \mathrm{kg}$ b.w in $1 \mathrm{ml}$ distilled water by oral gavage, 3 times a week for 14 weeks on opposite days of DMBA application), GIV : Oral administration of RA alone (100mg/kg b.w in $1 \mathrm{ml}$ distilled water by oral gavage, 3 times a week for 14 weeks).

Results: Gross observation and histopathological findings revealed a-GI: normal stratified squamous epithelium b- GII: well and moderately differentiated SCC c-: GIII: revealed considerably reversed tumor incidence d: GIV: normal similar to GI. Immunohistochemical results revealed, oral administration of RA to DMBA treated hamsters restored the normal expression of bcl2- and Bax indicating that RA may have chemopreventive mechanism and/or pro-apoptotic effects.
\end{abstract}

Conclusion: RA may act through different mechanisms, such as applying anti-inflammatory, antioxidant results as well as impede cell proliferation, migration, and selectively convince cancer cells apoptosis.

KEYWORDS: HBP carcinoma, rosmarinic acid, apoptosis.

\footnotetext{
* Lecturer, Oral and Dental Pathology Department, Faculty of Dental Medicine, (Boys- Cairo), Al-Azhar University, Egypt. ** Assistant Professor; Department of Oral Biology, Faculty of Dental Medicine, (Boys- Cairo), Al-Azhar University, Egypt.
} 


\section{INTRODUCTION}

Head and neck cancer is the sixth most widespread cancers recording approximately 600,000 new cases yearly globally ${ }^{(1)}$. Oral squamous cell carcinoma (OSCC) is the crucial subtype of head and neck cancer and accounts for two-thirds of the cases happen in least developed countries ${ }^{(2)}$. The National Cancer Registry Program of Egypt (NCRPE) reported the national incidence rates of cancer in 2015 for all sites excluding non-melanoma skin cancer as 113.1/100,000 (both sexes), 115.7/100,000 (males), and 110.3/100,000 (females) ${ }^{(3)}$. Oral cancer accounts for nearly $3 \%$ of all cancer cases ${ }^{(4)}$. Frequency of oral cancer in Egypt, (lip, tongue and mouth, excluding the tonsils and oropharynx) in 2015 was $0.9 \%$ in males and $0.75 \%$ in females ${ }^{(3)}$. More than $90 \%$ of oral cancer is $\operatorname{OSCC}^{(5,6)}$. OSCC is a multifactorial disease with many risk factors involved in its development, such as tobacco and alcohol consumption, human papilloma virus (HPV), nutritional deficiencies, immunosuppression, family predisposition and genetic instability ${ }^{(6,7)}$. OSCC can be presented as a "natural history", which originates from atypical keratinocytes which are persistent exposed to a stimulus that disturb its homeostasis, following an epithelial hyperplasia, dysplasia in varying degrees, carcinoma in situ and an invasive carcinoma leading to production of distant metastases ${ }^{(8)}$. When squamous epithelium is affected by multiple genetic changes oral carcinogenesis which is a highly complex multifocal process takes place ${ }^{(9)}$. Presumably oral carcinogenesis begin with the alteration of a limited number of typical keratinocytes through cytogenetic alterations and epigenetic processes that alter the development of the cell cycle, DNA repair mechanisms, cell differentiation and apoptosis ${ }^{(10)}$. DMBA is frequently used as a major carcinogen to evolve tumors in the golden Syrian $\mathrm{HBP}^{(11)}$. Buccal pouches, a pocket like anatomy, exposed to repeated topical applications of DMBA resulted in welldeveloped OSCC. DMBA produces neoplasms by inducing severe inflammation and dysplasia in the buccal pouches as well as by causing great DNA oxidative damage ${ }^{(12)}$. Multiple evidences indicate histological, morphological, biochemical and molecular similarities between DMBA induced oral tumors and human oral tumors ${ }^{(8)}$.

Plants and herbs are origin of compounds with prospective anticancer activity that are able to arrest, reverse and/or retard carcinogenesis at various stages. Among these compounds, polyphenols with pleiotropic properties that select different inflammatory, redox-sensitive and energysensing metabolic pathways by modulating the activity of different transcription factors, which is consistent with multifactorial character of cancer $^{(13,14)}$. Rosemary (Rosmarinus officinalis L.) is a woody plant of the Lamiaceae family that is mainly spread in the Mediterranean area. Exploratory researches has established chemoprotective effects against hepatotoxicity ${ }^{(15)}$, gastric ulcerative disease, and anticancer ${ }^{(16-19)}$ both in vitro and in vivo.

Diterpenes carnosic acid and rosmarinic acid (RA) are the main polyphenols found in rosemary extract (18). RA have a therapeutic role, with respect to its anti-oxidant, and anti-inflammatory activities $^{(20)}$. Pervious literature had described the growth inhibitory and anti-invasion properties of RA in colon, skin, breast and ovarian cancers in vitro and/or in vivo but the mechanisms underlying these effects are poorly understood ${ }^{(21-24)}$. It applies antiproliferative activity via induction of cell cycle arrest in G0/G1 and G1/S phase and induces apoptosis in cancer cells via mitochondrial pathway. Moreover, it has antiangiogenic activity ${ }^{(25,26)}$.

Among different factors, apoptosis, or programmed cell death, was found to play a pivotal task in the regulation of $\mathrm{OSCC}^{(27)}$. Since B cell lymphoma-2 (Bcl-2) is anti-apoptotic protein, it is expected to function as oncogenes in cancer cells and pro-apoptotic proteins such as $\mathrm{Bcl}-2$ Associated X Protein (Bax) is expected to act as tumor-suppressors. According to these postulations, cancers over-expressing Bcl-2 should have bad 
prognosis, while tumors over-expressing Bax should be associated with better clinical outcomes. nevertheless, researches survey the prognostic importance of apoptotic proteins in OSCC have submit conflicting results ${ }^{(28)}$.

Hence, the main target of the present study was to assess effect of RA as a chemopreventive modality on DMBA induced HBP carcinoma. Assessment was based on gross observation, histological changes of tumor tissue and immunohistochemical (IHC) examination utilizing Bcl-2 and Bax antibodies.

\section{MATERIAL AND METHODS}

Chemicals: DMBA $(0.5 \%)$ was obtained from Sigma-Aldrich Company, dissolved in paraffin oil. RA was obtained from Sigma-Aldrich Company.

Animals: forty Syrian male hamsters five weeks old, weighing 80-120g were achieved from the animal house, Cairo University (Cairo, Egypt). Experimental animals were sheltered in standard cages with sawdust bedding under controlled environmental circumstances of humidity (30-40\%), temperature $\left(20 \pm 2^{\circ} \mathrm{C}\right)$, and light (12-h light/12-h dark). All experimental animals were supplied with standard diet and water ad libitum.

Experimental design: The experimental hamsters were categorized into 4 groups of 10 animals in each as follow: GI: Topical application of liquid paraffin alone (3 times a week for 14 weeks),GII: Topical application of DMBA alone, the rights HPB were painted with $0.5 \%$ DMBA in paraffin oil using a number 4 camel hair brush 3 times a week for 14 weeks, GIII: Topical application of DMBA $(0.5 \%$ in liquid paraffin, 3 times a week for 14 weeks) associated by oral administration of RA $(100 \mathrm{mg} / \mathrm{kg}$ b.w in $1 \mathrm{ml}$ distilled water by oral gavage ${ }^{(23)}, 3$ times a week for 14 weeks on opposite days of DMBA application), GIV : Oral administration of RA alone $(100 \mathrm{mg} / \mathrm{kg}$ b.w in $1 \mathrm{ml}$ distilled water by oral gavage, 3 times a week for 14 weeks).
Following termination of the experiment, all animals were euthanized, then hamster's head were separated and fixed in 10\% buffered neutral formalin solution for $24 \mathrm{~h}$ after putting a piece of wood with suitable size, between hamster's teeth to prevent a wrinkling or sloughing of the hamster's mucosa during manipulation and preparation. Using a bard-parker scalpel, no 15, small surgical scissors and tweezers, the specimens were excised from right HPB mucosa and trimmed to 1-2 $\mathrm{cm}$ average size. Tissue specimens were excised, then processed for hematoxylin and eosin H\&E and IHC staining.

Histopathological examinations: Sections of $3-5 \mu \mathrm{m}$ thickness were cut utelizing a rotary microtome and stained with $\mathrm{H} \& \mathrm{E}$ dye for microscopic examination.

Immunohistochemical examinations: Other tissue sections were cut for the application of standard labeled streptavidin- biotin method to demonstrate the expression of Bcl-2 \& Bax antibodies. Paraffin embedded tissue sections were dewaxed and rehydrated through graded ethanol to distilled water. Endogenous peroxidase was blocked by incubation with $3 \% \mathrm{H}_{2} \mathrm{O}_{2}$ in methanol for $10 \mathrm{~min}$. Antigen retrieval was acquired by adding citrate buffer solution ( $\mathrm{pH}$ 6.0) and put in microwave for 3 intervals, 5 min each at $95^{\circ} \mathrm{C}$, followed by washing with phosphate buffered saline (PBS). Tissue sections were then received one or two drops of primary antibodies (Bcl-2 \& Bax) in a dilution of 1:100 in Tris buffer solution and incubated in a humid chamber at room temperature overnight at $4^{\circ} \mathrm{C}$. After washing with PBS, Biotinylated secondary antibody was added and incubated for $30 \mathrm{~min}$ at room temperature. After rinsing with PBS, tissue sections were received diaminobenzidine (Sigma, USA) was applied for 2-4 min to develop color. When acceptable color intensity acquired, the slides were washed, counter stained with hematoxylin and covered with a mounting medium.

Immunostained sections were examined using light microscope to evaluate the prevalence of 
positive cases and localization of immunostaining within the tissues. In addition, image analysis computer system was used to assess area percentage of positive cells of immunostaining.

This was done in Oral and Dental Pathology Department - Faculty of Dental Medicine - BoysCairo - Al-Azhar University. The degree of positive staining for antibody was evaluated by a wellestablished semi-quantitative scoring on a scale range from negative to strong positive staining as follow: Strong staining (more than 50\% stained), moderate staining (between 25 and 50\% stained), weak staining (between 5 and $25 \%$ stained), and negative (less than $5 \%$ stained). ${ }^{(29)}$

Statistical analysis Results are expressed as the mean \pm standard deviation (SD) and are accompanied by the number of observations. A oneway analysis of variance (ANOVA) was performed using SPSS version 17.0 for windows. The results were considered statistically significant when the probability was $<0.05$.

\section{RESULTS}

Gross observation: HBP mucosa of GI were pink in color with smooth surface with no observable abnormalities (Fig.1A). In GII, HBP mucosa showed multiple exophytic nodules of variable size.
The nodules were surrounded with ulcerative and bleeding areas. Depilating of all animals was an observable remark (Fig.1B). In GIII, HBP mucosa showed variable changes included erythematous mucosal surface to normal color with smooth surface and the animals appeared healthy (Fig.1C). In GIV, HBP mucosa showed normal appearance similar to GI (Fig.1D).

Histopathological and immunohistochemical results: The tissue sections of HBP mucosa of experimental groups showed variable results in regard to the histopathological results and IHC results. In GI, histological sections, using H\&E stain, revealed normal HBP mucosa, composed of thin stratified squamous epithelium, consists of 2 to 4 layers of squamous cells showing slight keratinization (i.e.; one layer of basal cells and one, two or three layers of spinous and thin keratinized cells with abscent rete ridges. Sub epithelial connective tissue (C.T), muscular layer and areolar layer were seen (Fig.2A). IHC staining using Bcl2 antibody exhibited weak positive expression (mean $=6.5)$ which limited to basal and suprabasal layers (Fig.2B) while Bax expression showed moderate positive expression $($ mean $=48.12)$ which present throughout the epithelial layers (Fig.2C). In GII, histological sections, using H\&E stain, 5 animals exhibited well differentiated SCC and 5 animals exhibited moderately differentiated SCC.

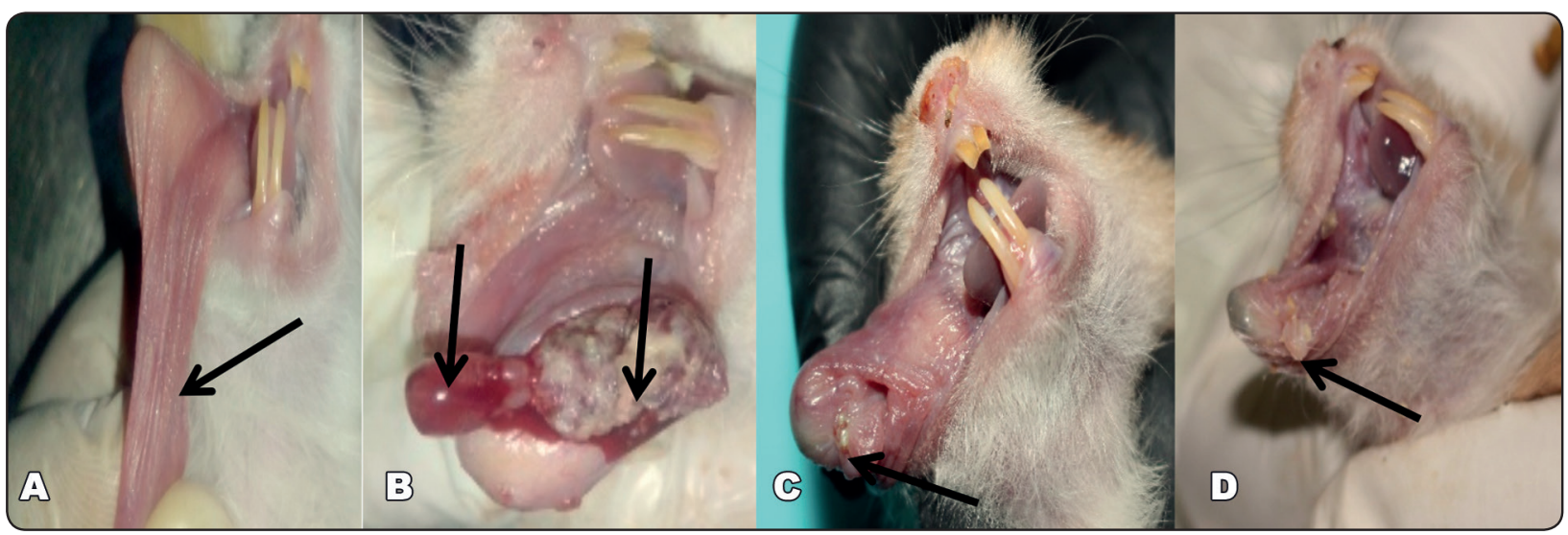

Fig. 1 (A): HBP of GI showing normal buccal pouch mucosa which appeared pink in color with smooth surface (arrow). Fig. 1(B): HBP of GII showing multiple exophytic nodules surrounded with ulcerative and bleeding areas (arrows). Fig. 1 (C): HBP of GIII showing variable changes included erythematous mucosal surface to normal color with smooth surface (arrow). Fig.1(D): HBP of GIV showing normal appearance (arrow). 
Dysplastic features in multiple areas, destruction of basement membrane, and prominent true invasion with formation of various forms of epithelial nests (Fig.2D). IHC staining using Bcl-2 exhibited strong positive cytoplasmic expression $($ mean $=75.16)$ throughout the tumor cells (Fig.2E) while Bax exhibited weak positive cytoplasmic expression $($ mean $=13.56)$ throughout the tumor cells $($ Fig.2F). In GIII, histological sections, using H\&E stain, 2 out of 10 animals exhibited mild epithelial dysplasia and 8 out of 10 animals exhibited hyperkeratosis to normal epithelium (Fig.2G). IHC staining using Bcl-2 exhibited moderate positive cytoplasmic expression $($ mean $=27.13)$ throughout the epithelial layers (Fig.2H) while Bax expression showed moderate positive cytoplasmic expression (mean = 45.22) throughout the epithelial layers (Fig.2I). In GIV, histological sections using H\&E stain and IHC staining using Bcl-2 and Bax expression was similar that of GI.

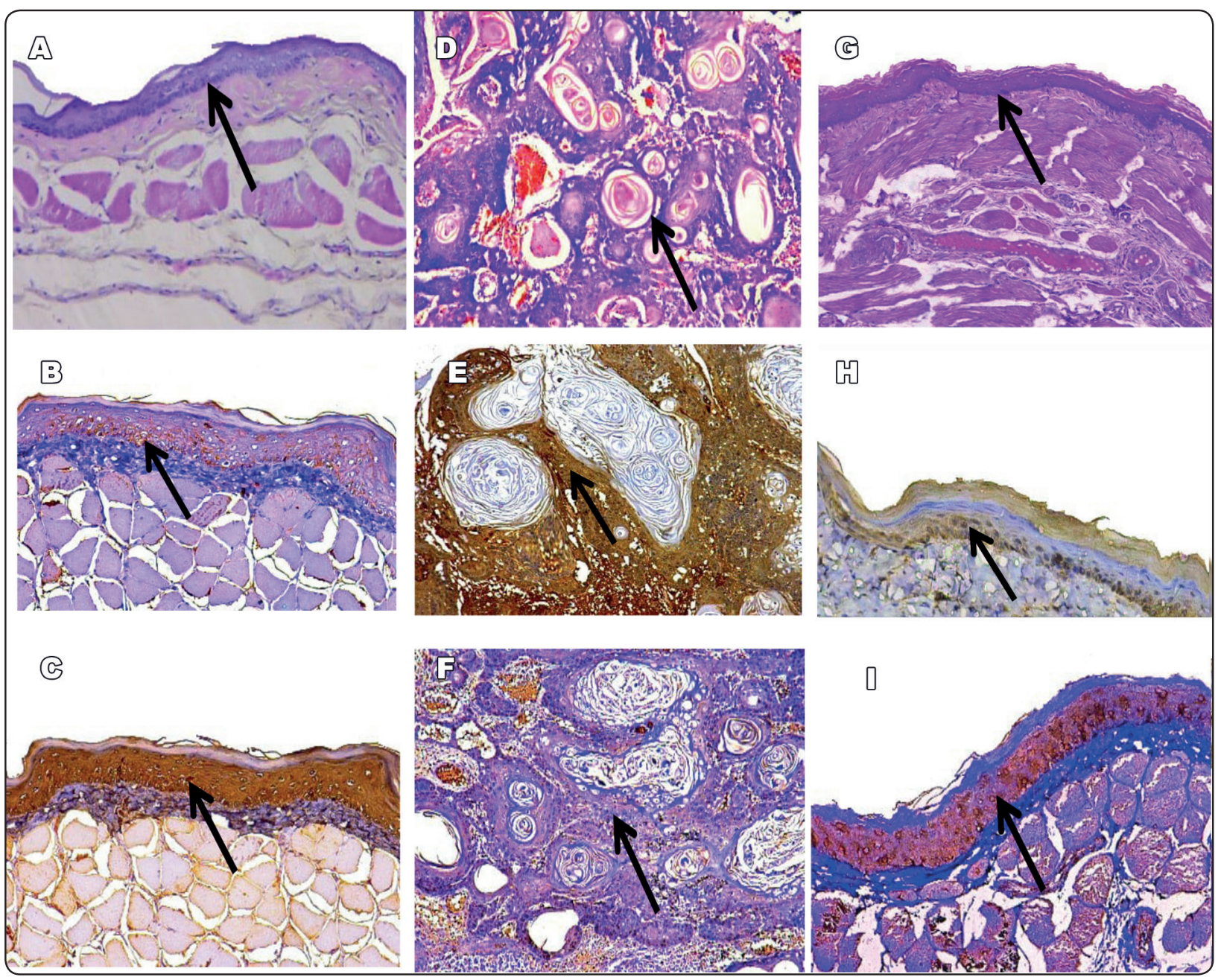

Fig. 2 (A): H\&E stain of GI showing keratinized stratified squamous epithelium with flattened rete ridges, sub-epithelial C.T layer and muscular layer (X200). Fig.2(B): IHC expression of Bcl-2 showing positive cytoplasmic expression in basal and suprabasal epithelial layers (X200). Fig.2(C): IHC expression of Bax showing positive cytoplasmic expression throughout the epithelial layers (X200). Fig.2(D): H\&E stain of GII of HBP mucosa showing well differentiated SCC (X200). Fig.2(E): IHC expression of Bcl-2 showing positive cytoplasmic expression throughout the tumor cells (X200). Fig.2(F): IHC expression of Bax showing positive cytoplasmic expression throughout the tumor cells (X200). Fig.2(G): H\&E stain of GIII of HBP mucosa showing mild epithelial dysplasia (X200). Fig.2(H): IHC expression of Bcl-2 showing positive cytoplasmic expression throughout the epithelial layers (X200). Fig.2(I): IHC expression of Bax showing positive cytoplasmic expression throughout the epithelial layers (X200). 
Statistical analysis results of Bcl-2 \& Bax expression were obtained by comparing the area $\%$ of Bcl-2 \& Bax expression in the groups used. Statistical analysis results were revealed that, In regard to expression of Bcl-2, GI had recorded the lowest mean area percentage (6.5\%), while GII had the highest mean area percentage $(75.16 \%)$ while in regard to expression of Bax, GII had recorded the lowest mean area percentage $(13.56 \%)$, while GI had the highest mean area percentage $(48.12 \%)$. Comparing the DMBA treated group (GII) with GI (normal) \& GIV, there was highly significant difference regarding $\mathrm{Bcl}-2$ and $\mathrm{Bax}$; the $\mathrm{P}$ value recorded $0.001(<0.01)$. Comparing the GIII with GI (normal) \& GIV, there was highly significant difference regarding $\mathrm{Bcl}-2$, the $\mathrm{P}$ value recorded $0.005(<0.01)$ while Bax shown non-significant difference between the same groups, the $\mathrm{P}$ value recorded 0.441 ( $>0.05)$. Comparing the GIII with GII, there was highly significant difference regarding Bcl-2 and Bax; the P value recorded $0.001(<0.01)$.

TABLE (1)

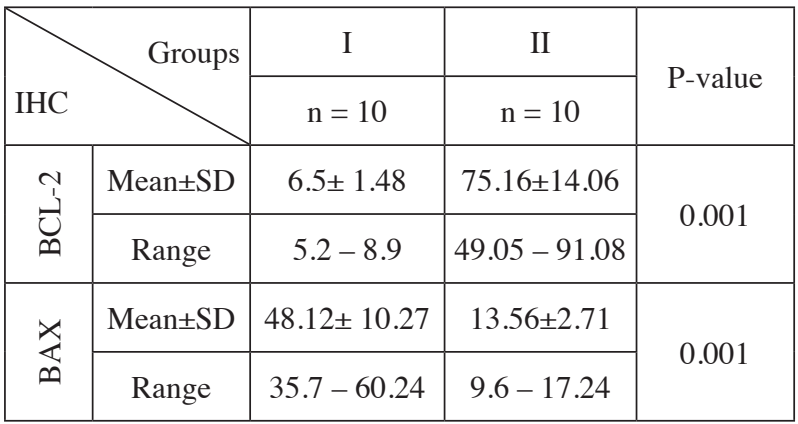

TABLE (2)

\begin{tabular}{|c|c|c|c|c|}
\hline \multirow{2}{*}{\multicolumn{2}{|c|}{ IHC groups }} & I & III & \multirow{2}{*}{ P-value } \\
\hline & & $\mathrm{n}=10$ & $\mathrm{n}=15$ & \\
\hline \multirow{2}{*}{ 己ֶ } & Mean \pm SD & $6.5 \pm 1.48$ & $27.13 \pm 6.83$ & \multirow{2}{*}{0.005} \\
\hline & Range & $5.2-8.9$ & $22.17-32.93$ & \\
\hline \multirow{2}{*}{$\underset{\infty}{x}$} & Mean \pm SD & $48.12 \pm 10.27$ & $45.22 \pm 9.28$ & \multirow{2}{*}{0.441} \\
\hline & Range & $35.7-60.24$ & $37.51-57.18$ & \\
\hline
\end{tabular}

TABLE (3)

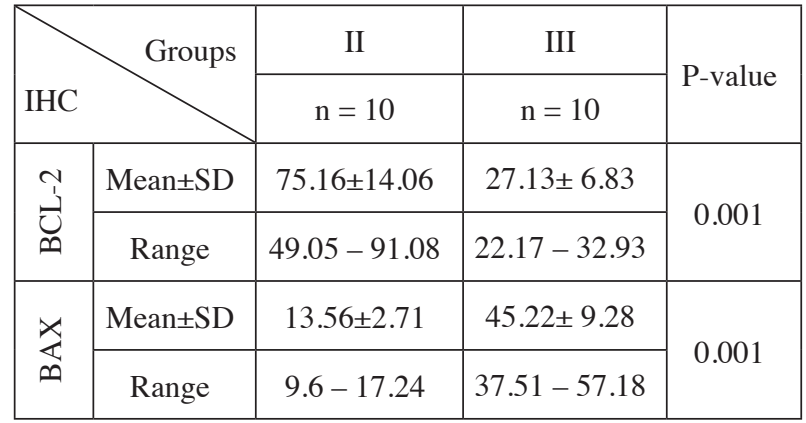

Bar chart representing mean area \% results of $B C l-2 \&$ Bax between GI, GII, GIII and GIV

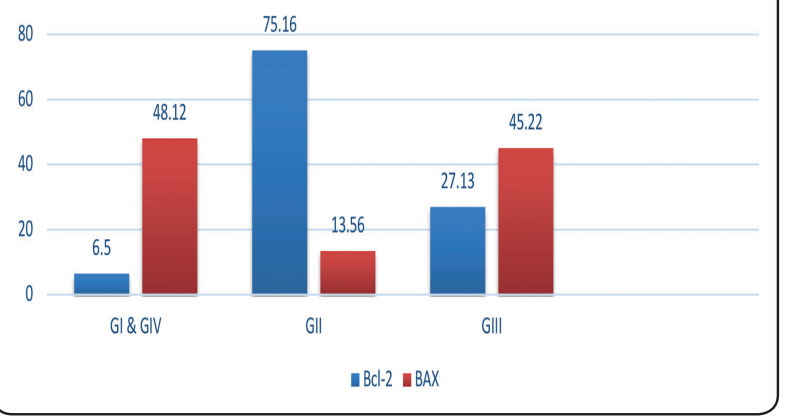

\section{DISCUSSION}

Oral carcinogenesis has been widely experimented, point to progress either biomarkers for early diagnosis or successful treatment. HBP model is effective due to resemblance to human oral carcinogenesis at both histological and molecular level. Moreover, DMBA was chosen as the chemical carcinogen, because it plays the same etiological role in hamster SCC as do alcohol and tobacco in human OSCC. In this study, we evaluate the effect of RA as a chemopreventive modality on experimentally induced HBP carcinogenesis. The results of gross observations, $H \& E$ stain and IHC staining utilizing Bcl-2 and Bax antibodies, revealed variable observations.

In the present study, GI (normal group), the gross observation showed no observable changes. HBP mucosa appeared normal, with smooth surface and the buccal pouch length was about $5 \mathrm{~cm}$. 
This finding reflected on H\&E staining which showed thin keratinized stratified squamous epithelium. Subepithelial C.T formed of small amount of sporadic fibrocytes and blood vessels which present between the epithelium and the muscular layer. Bruna et al, 2017(30) reported the same finding. In the current study, GI (normal group), IHC results of Bcl-2 showed weak positive expression (mean $=6.5$ ) which limited to basal and suprabasal layers while Bax expression showed moderate positive expression (mean $=48.12$ ) which present throughout the epithelial layers. The finding of this study was in agreement of some researches ${ }^{(31-34)}$. Karthikeyan et al, 2014 ${ }^{(35)}$ stated that, under usual circumstances, $\mathrm{Bcl}-2 / \mathrm{Bax}$ correlation determines the fate of cell survival or cell death, through regulation of release of cytochrome $\mathrm{C}$ from the mitochondria. This result may be due to that; $\mathrm{Bcl}-2$ assist in the control of the terminal differentiation of keratinocytes by secured their stem cells from apoptosis.

In the current study, GII (DMBA painted group) at 14 weeks, gross observation showed multiple large exophytic nodules surrounded with ulcerative and bleeding areas, some animals appeared debilitated. H\&E stain, showed a development of well and moderately differentiated SCC. This is in consistence with that shown by other studies ${ }^{(36,37)}$. This observations might be attributed to DMBA effect on metabolic activation, DMBA is converted into its active metabolic carcinogen, dihydrodiol epoxide, which mediates carcinogenesis through overproduction of ROS, chronic inflammation, activation of protoncogene, deactivation of tumor suppressor genes, extensive DNA damage, and reduction in DNA repair which reduced the ability to induce apoptosis ${ }^{(38,39)}$. The current results supported the concept that DMBA induced HBP carcinoma appeared to go through the same changes as in human not only at gross observations but also at light microscopic observations. These results are in accordance with those of previous studies ${ }^{(40-42)}$.
In this study, oral administration a dose of $100 \mathrm{mg} / \mathrm{kg}$ body wt of RA not only completely prevented the formation of oral tumors, but also markedly reduces preneoplastic lesions severity such as hyperplasia, keratosis, and dysplasia in DMBA-treated hamsters. Our results indicate that RA has vigorous efficacy in prevention of oral carcinogenesis (chemopreventive capability). These findings conflicted on H\&E staining in which only 2 animals exhibited mild epithelial dysplasia and 8 animals exhibited hyperkeratosis to normal. These findings are in line with Anusuya et al, 2011 ${ }^{(43)}$ they notice that, phase I liver detoxification enzymes were considerably increased, while phase II enzymes were decreased in hamsters treated with DMBA alone. Liver plays a critical role in detoxification and metabolic activation of carcinogenic agents. The altered condition of phase I and phase II agents in the liver of DMBA treated animals suggests that liver activity was significantly weakned during DMBA-induced oral carcinogenesis. Increased activities of phase I enzymes in liver suggest that DMBA metabolized actively during carcinogenesis. Decreased activities of phase II enzymes in liver suggest that functions of these enzymes are impaired due to aggregation of carcinogenic metabolites in liver or impairment in conjugation or detoxification of carcinogenic metabolites during DMBA induced oral carcinogenesis. Status of phase I and phase II detoxification enzymes in the liver of DMBA-treated hamsters returned to near normal range by oral administration of RA at a dose of $100 \mathrm{mg} / \mathrm{kg}$ body wt. Their results suggest that metabolic activation of DMBA or stimulation the process of conjugation of carcinogenic metabolites with glutathione as well as stimulation the excretion of carcinogenic metabolites of DMBA may be inhibited by RA. These observation are in agreement with those of other studies ${ }^{(44,45)}$.

In the current study, hamsters treated with DMBA alone had overexpression of Bcl-2 and down expression of Bax. IHC analysis of Bcl-2 was $75.16 \%$ 
positive staining while IHC analysis of Bax was $13.56 \%$ in hamsters treated with DMBA alone. Oral administration of RA to DMBA-treated hamsters suppressed the expression of Bcl-2 (27.13\%) and increased the expressions of Bax (45.22\%). These findings advocate that RA might have inhibited abnormal cell proliferation either by inhibiting DMBA metabolic activation or by suppressing DMBA-induced mutations in Bcl-2 during DMBAinduced oral carcinogenesis. These results are in agreement with those of other investigators ${ }^{32,} 46$. 47). The increase of Bcl-2 expression indicated that inhibition of apoptosis by prevention the release of cytochrome $\mathrm{C}$ from mitochondria and promotion of carcinogenesis, while the decreased expression of Bax could be due to reduced apoptotic cell death as well as accelerated their growth ${ }^{(33,37,41)}$.

There was highly statistically significant differences between the expression of $\mathrm{Bcl}-2$ and Bax in GII and GIII (the P value recorded 0.001) which mean, the increased expression of Bcl-2 and decreased expression of Bax in OSCC as compared to normal or oral epithelial dysplasia may be an evidence of the disease progression of oral epithelial dysplasia to OSCC, as the results of this study suggest variations in expression of Bcl-2 family proteins, creating a convenient environment for malignant transformation. The results of this work showed that, inhibition of apoptosis is a frequent event in oral carcinogenesis. Bcl-2 family proteins appears to be involved in regulating the terminal differentiation of keratinocytes. Down and up regulation of Bcl-2 and Bax expressions were associated with terminal differentiation. Over expression of antiapoptotic protein Bcl-2 and down expression of proapoptotic protein Bax, preserve tumor cells from undergoing apoptosis, thus assisting their survival.

The current study express chemopreventive potential of RA in DMBA-induced hamster buccal pouch carcinogenesis. However the chemopreventive mechanism of RA is uncertain. Anusuya et al, 2011 ${ }^{(43)}$ attributed chemopreventive mechanism to antilipid peroxidative potential and modulating effect on detoxification cascades of Bcl-2 expression could play a probable role. This results are in line with Sharmila et al, 2012 ${ }^{(23)}$ whom observed that, over expression of antiapoptic Bcl2 and downexpression of proapoptic Bax observed in the skin tumor tissues of DMBA painted animals propose evasion of apoptosis and abnormal cell proliferation. Results of the study showed disability of apoptotic machinery in mice treated with DMBA alone. RA oral administration to DMBA treated mice repair the expression of $\mathrm{p} 53, \mathrm{Bcl}-$ 2, Bax, caspase-3 and 9, indicating that RA may have facilitate or motivate the process of apoptosis during DMBA induced skin carcinogenesis. Cao et al, 2019 ${ }^{(48)}$ attributed chemopreventive mechanism to RA exerts pro-apoptotic effects by inhibiting of STAT3 pathway, thereby upregulating Bax and down-regulating Bcl-2. Nadeem et al, 2019(49) suggested that, RA's anti-angiogenic effects, as demonstrated through human umbilical vein endothelial cells proliferation, migration, adhesion, and tube formation inhibition. Exact mechanistic pathway for the chemopreventive potential of RA by analyzing more molecular markers that have a potent role in carcinogenesis need further studies.

\section{CONCLUSIONS}

Present evidence advocate exploration of RA as a hopeful therapeutic agent against a wide variety of modernistic lifestyle disorder. However, mechanisms underlying RA's therapeutic efficacy need moreover examinations. Different researches showed that RA may act through numerous mechanisms, such as exerting anti-inflammatory and antioxidant effects in addition to inhibiting cell proliferation, migration, and inducing cancer cells apoptosis. Furthermore, RA's anti-angiogenic effects, as explained through human umbilical vein endothelial cells proliferation, migration, adhesion, and tube formation inhibition, propose that it can be valuable in inhibiting tumor growth and metastasis. 


\section{RECOMMENDATIONS}

Rosemary extract can be considered as a rich source of potential candidates to be included in the diet with favorable effects at pre-evaluated doses, avoiding toxicity.

\section{REFERENCES}

1. Ferlay J, Soerjomataram I, Dikshit R, Eser S, Mathers C, Rebelo M, et al. Cancer incidence and mortality worldwide: sources, methods and major patterns in GLOBOCAN 2012. Int J Cancer. 2015;136(5):E359-86.

2. Jemal A. Global burden of cancer: opportunities for prevention. Lancet. 2012; 380(9856):1797-99.

3. Shaaban N, Raslan H, Ramadan O, Habib A, Zaki E. Micronuclei as biomarkers of genetic damage in oral squamous cell carcinoma. Alexa Dent J. 2018;43(3):28-33.

4. Ferlay J, Shin H, Bray F, Forman D, Mathers C, Parkin D. Estimates of worldwide burden of cancer in 2008: GLOBOCAN 2008. Int J Cancer 2010;127(12):2893-917.

5. Organization WH. The global burden of disease: 2004 update. 2008.

6. Feller L, Lemmer J. Oral squamous cell carcinoma: epidemiology, clinical presentation and treatment. J Cancer Ther. 2012;3(4):263- 68.

7. Radoï L, Luce D. A review of risk factors for oral cavity cancer: the importance of a standardized case definition. Community Dent Oral Epidemiol. 2013;41(2):97-109.

8. Tanaka T, Ishigamori R. Understanding carcinogenesis for fighting oral cancer. J Oncol. 2011;2011:603740.

9. Joseph B. Oral cancer: prevention and detection. Med Princ Pract. 2002;11(1):32-35.

10. Feller L, Khammissa R, Kramer B, Lemmer J. Oral squamous cell carcinoma in relation to field precancerisation: pathobiology. Cancer Cell Int. 2013; 13(1):31-39.

11. Karthikeyan S, Srinivasan R, Wani S, Manoharan S. Chemopreventive potential of chrysin in 7, 12-dimethylbenz (a) anthracene-induced hamster buccal pouch carcinogenesis. Int J Nutr Pharmacol Neurol Dis. 2013;3(1):46-53.

12. Manoharan S, Karthikeyan S, Essa M, Manimaran A, Selvasundram R. An overview of oral carcinogenesis. Int J Nutr Pharmacol Neurol Dis. 2016;6(2):51-62.
13. Barrajón E, Herranz M, Joven J, Segura A, Alonso C, Menéndez J, et al. Molecular promiscuity of plant polyphenols in the management of age-related diseases: far beyond their antioxidant properties Adv Exp Med Biol. 2014;824:141-59.

14. Menendez J, Joven J, Aragonès G, Barrajón E, Beltrán $\mathrm{R}$, Borrás I, et al. Xenohormetic and anti-aging activity of secoiridoid polyphenols present in extra virgin olive oil: a new family of gerosuppressant agents. Cell Cycle. 2013;12(4):555-78.

15. Rašković A, Milanović I, Pavlović N, Ćebović T, Vukmirović S, Mikov M. Antioxidant activity of rosemary (Rosmarinus officinalis L.) essential oil and its hepatoprotective potential. BMC Complement Altern Med. $2014 ; 14: 225$.

16. Aoki A, Akaboshi H, Ogura T, Aikawa T, Kondo T, Tobori $\mathrm{N}$, et al. Preparation of $\mathrm{pH}$-sensitive anionic liposomes designed for drug delivery system (DDS) application. J Oleo Sci. 2015;64(2):233-42.

17. Arai T, Benny O, Joki T, Menon L, Machluf M, Abe T, et al. Novel local drug delivery system using thermoreversible gel in combination with polymeric microspheres or liposomes. Anticancer Res. 2010;30(4):1057-64.

18. Moore J, Yousef M, Tsiani E. Anticancer effects of rosemary (Rosmarinus officinalis L.) extract and rosemary extract polyphenols. Nutrients. 2016;8(11):731.

19. Borrás I, Pérez A, Lozano J, Barrajón E, Arráez D, Cifuentes $\mathrm{A}$, et al. A bioguided identification of the active compounds that contribute to the antiproliferative/cytotoxic effects of rosemary extract on colon cancer cells. Food Chem Toxicol.2015;80:215-22.

20. Tumur Z, Guerra C, Yanni P, Eltejaye A, Waer C, Alkam $\mathrm{T}$, et al. Rosmarinic acid inhibits cell growth and migration in head and neck squamous cell carcinoma cell lines by attenuating epidermal growth factor receptor signaling. J Cancer Sci Ther. 2015;7(12):367-74.

21. Xu Y, Xu G, Liu L, Xu D, Liu J. Anti-invasion effect of rosmarinic acid via the extracellular signal-regulated kinase and oxidation-reduction pathway in Ls174-T cells. J Cell Biochem. 2010;111(2):370-79.

22. Scheckel K, Degner S, Romagnolo D. Rosmarinic acid antagonizes activator protein-1-dependent activation of cyclooxygenase- 2 expression in human cancer and nonmalignant cell lines. J Nutr. 2008;138(11):2098-105. 
23. Sharmila R, Manoharan S. Anti-tumor activity of rosmarinic acid in 7, 12-dimethylbenz (a) anthracene (DMBA) induced skin carcinogenesis in Swiss albino mice. Indian J Exp Biol. 2012;50(3):187-94.

24. Xu Y, Jiang Z, Ji G, Liu J. Inhibition of bone metastasis from breast carcinoma by rosmarinic acid. Planta Med. 2010;76(10):956-62.

25. Wu C, Hong C, Klauck S, Lin Y, Efferth T. Molecular mechanisms of rosmarinic acid from Salvia miltiorrhiza in acute lymphoblastic leukemia cells. J Ethnopharmacol. 2015;176:55-68.

26. Hashiesh H, Elkhoely A, Eissa A, Youns M. Rosmarinic acid enhances cisplatin cytotoxicity in Hepg2 cell line and attenuates its nephrotoxicity in mice. IJPSR. 2018;9:273143.

27. Muzio L, Sartini D, Santarelli A, Rocchetti R, Morganti $\mathrm{S}$, Pozzi V, et al. Expression and prognostic significance of apoptotic genes in oral squamous cell carcinoma. Mol Carcinog. 2014;53(4):264-71.

28. Bose P, Klimowicz A, Kornaga E, Petrillo S, Matthews T, Chandarana $\mathrm{S}$, et al. Bax expression measured by AQUAnalysis is an independent prognostic marker in oral squamous cell carcinoma. BMC cancer. 2012;12(1):332-44.

29. Negi A, Puri A, Gupta R, Nangia R, Sachdeva A, Mittal M. Comparison of immunohistochemical expression of antiapoptotic protein survivin in normal oral mucosa, oral leukoplakia, and oral squamous cell carcinoma. Patholog Res Int. 2015;2015:840739.

30. Bruna F, Arango M, Plaza A, Espinoza I, Conget P. The administration of multipotent stromal cells at precancerous stage precludes tumor growth and epithelial dedifferentiation of oral squamous cell carcinoma. Stem Cell Res. 2017;18:5-13.

31. Camisasca D, Honorato J, Bernardo V, Silva L, Fonseca E, Faria P, et al. Expression of Bcl-2 family proteins and associated clinicopathologic factors predict survival outcome in patients with oral squamous cell carcinoma. Oral Oncol. 2009;45(3):225-33.

32. Rajasekaran D, Manoharan S, Silvan S, Vasudevana K, Baskaran N, Palanimuthu D. Proapoptotic, anti-cell proliferative, anti-inflammatory and antiangiogenic potential of carnosic acid during 7, 12 dimethylbenz [a] anthraceneinduced hamster buccal pouch carcinogenesis. Afr J Tradit Complement Altern Med. 2012;10(1):102-12.
33. Manoharan S, Sindhu G, Nirmal M, Vetrichelvi V, Balakrishnan S. Protective effect of berberine on expression pattern of apoptotic, cell proliferative, inflammatory and angiogenic markers during 7,12-dimethylbenz (a) anthracene induced hamster buccal pouch carcinogenesis. Pak J Biol Sci. 2011;14(20):918-32 .

34. Ribeiro D, Salvadori D, Marques M. Abnormal expression of bcl-2 and bax in rat tongue mucosa during the development of squamous cell carcinoma induced by 4-nitroquinoline 1-oxide. Int J Exp Pathol. 2005; 86(6):375-81.

35. Karthikeyan S, Manoharan S. Cromolyn inhibits 7, 12-dimethylbenz (a) anthracene induced oral cancer through apoptotic induction and suppression of cell proliferation. Int J Pharm Bio Sci. 2016; 7(1):35-42.

36. Sophia J, Kowshik J, Mishra R, Nagini S. Nimbolide, a neem limonoid inhibits phosphatidyl inositol-3 kinase to activate glycogen synthase kinase- $3 \beta$ in a hamster model of oral oncogenesis. Sci Rep. 2016 Feb 23;6(1):1-13.

37. Manoharan S, Rajasekaran D, Prabhakar M, Karthikeyan S, Manimaran A. Modulating effect of Enicostemma littorale on the expression pattern of apoptotic, cell proliferative, inflammatory and angiogenic markers during 7 , 12-dimethylbenz (a) anthracene induced hamster buccal pouch carcinogenesis. Toxicol Int. 2015;22(1):130-40.

38. Manoharan S, Wani S, Vasudevan K, Manimaran A, Prabhakar M, Karthikeyan S, et al. Saffron reduction of 7, 12-dimethylbenz [a] anthracene-induced hamster buccal pouch carcinogenesis. Asian Pac J Cancer Prev. 2013;14(2):951-57.

39. Murata M, Ohnishi S, Seike K, Fukuhara K, Miyata N, Kawanishi S. Oxidative DNA damage induced by carcinogenic dinitropyrenes in the presence of P450 reductase. Chem Res Toxicol. 2004;17(12):1750-56.

40. Silvan S, Manoharan S. Apigenin prevents deregulation in the expression pattern of cell-proliferative, apoptotic, inflammatory and angiogenic markers during 7, 12-dimethylbenz [a] anthracene-induced hamster buccal pouch carcinogenesis. Arch Oral Biol. 2013;58(1):94-101.

41. Nagini S, Letchoumy P, Thangavelu A, Ramachandran C. Of humans and hamsters: a comparative evaluation of carcinogen activation, DNA damage, cell proliferation, apoptosis, invasion, and angiogenesis in oral cancer patients and hamster buccal pouch carcinomas. Oral Oncol. 2009;45(6):e31-e7. 
42. Manoharan S, Palanimuthu D, Baskaran N, Silvan S. Modulating effect of lupeol on the expression pattern of apoptotic markers in 7, 12-dimethylbenz (a) anthracene induced oral carcinogenesis. Asian Pac J Cancer Prev 2012; 13(11):5753-57.

43. Anusuya C, Manoharan S. Antitumor initiating potential of rosmarinic acid in 7, 12-dimethylbenz (a) anthraceneinduced hamster buccal pouch carcinogenesis. J Environ Pathol Toxicol Oncol. 2011;30(3):199-211.

44. Manoharan S, Kavitha K, Senthil N, Renju G. Evaluation of anticarcinogenic effects of Clerodendron inerme on 7, 12-dimethylbenz (a) anthracene-induced hamster buccal pouch carcinogenesis. Singapore Med J. 2006; 47(12):1038-43.

45. Kavitha K, Manoharan S. Anticarcinogenic and antilipidperoxidative effects of Tephrosia purpurea (Linn.) Pers. in 7, 12-dimethylbenz (a) anthracene (DMBA) induced hamster buccal pouch carcinoma. Indian J Pharmacol 2006;38(3):185-89.
46. Kathiresan S, Govindhan A. [6]-Shogaol, a Novel chemopreventor in 7, 12-dimethylbenz [a] anthracene-induced hamster buccal pouch carcinogenesis. Phytother Res. 2016;30(4):646-53.

47. Subramaniyan J, Krishnan G, Balan R, Divya M, Ramasamy E, Ramalingam S, et al. Carvacrol modulates instability of xenobiotic metabolizing enzymes and downregulates the expressions of PCNA, MMP-2, and MMP-9 during diethylnitrosamine-induced hepatocarcinogenesis in rats. Mol Cell Biochem. 2014;395(1-2):65-76.

48. Cao W, Mo K, Wei S, Lan X, Zhang W, Jiang W. Effects of rosmarinic acid on immunoregulatory activity and hepatocellular carcinoma cell apoptosis in $\mathrm{H} 22$ tumor-bearing mice. Korean J Physiol Pharmacol. 2019;23(6):501-08.

49. Nadeem M, Imran M, Aslam T, Imran A, Shahbaz M, Amir $\mathrm{R}$, et al. Therapeutic Potential of Rosmarinic Acid: A Comprehensive Review. Appl Sci. 2019;9(15):3139. 